\title{
RUI: Recording user input from interfaces under Windows and Mac OS X
}

\author{
URMILA KUKREJA, WILLIAM E. STEVENSON, and FRANK E. RITTER \\ Pennsylvania State University, University Park, Pennsylvania
}

\begin{abstract}
Event and timing logs are useful in studying human-computer interaction, evaluating applications, and comparing input devices. Recording User Input (RUI) is a tool that records user-computer interface behavior. It is created in the .Net framework with C\# for Windows and in the Carbon framework for Mac OS X. RUI runs in the background and works with software that runs under Windows or Mac OS X (10.3 Panther and later versions). We illustrate its use with a human-robot interaction interface and present two simple tests that RUI passes and that other timing software should pass: avoiding 0 -msec timings and time distributions that follow a gamma (or gamma-like) distribution.
\end{abstract}

Timing records of the way users interact with applications can lead to useful insights into human behavior and an application's usability. Timing records can be useful for comparing different software applications and are also necessary for building and testing user models.

Westerman et al. (1996) described three different methods through which user information can be obtained. The first method, using videotape or a human recorder, is not always feasible. The video recording must be analyzed, and the human recorder has known limitations. Camtasia (TechSmith) is an example of a tool to record and replay user interactions as a video file. However, timing information cannot be directly obtained, and the tool creates logs that, being videos, are resource intensive.

The second method, instrumentation, is the augmentation of an interface so that it records the user's actions. This approach can be used only if the system studied includes logging or can be modified to include logging, so it is not a general solution; information collected in this way is limited to software that can be instrumented. For example, some commercial applications, such as Microsoft Word and many games, cannot be instrumented to record user behavior.

The third method is to include an unobtrusive application that exists in the background, can be used generically across all applications, and can record and timestamp user

This project was partially supported by ONR Contracts N00014-021-0021 and N00014-03-1-0248. The Windows version of RUI was part of U.K.'s master's thesis in computer science and engineering. Richard Carlson, Georg Jahn, Jong Kim, Diana Rösler, and two anonymous reviewers provided useful comments that improved this article. The software, including the source code, installation instructions (a README), example log files, and a movie of its application, is freely available at acs.ist.psu.edu/RUI. RUI for Windows requires that the .Net framework be installed. Details and the code for .Net are available at msdn .microsoft.com/netframework/downloads/framework1_1/. RUI for Mac OS X works without system modification. Correspondence concerning this article should be addressed to F. E. Ritter, College of Information Sciences and Technology, Pennsylvania State University, University Park, PA 16802 (e-mail: frank.ritter@psu.edu). behavior. In this article, we introduce such a program: Recording User Input (RUI), which belongs to the set of programs known as keystroke logging tools. Several such tools currently exist, but there are few that record both mouse and key events and even fewer that give logs in a form that would be useful to researchers and practitioners of human-computer interaction. Several of these recording and playback tools can be downloaded from the Web, but typically these were developed as malicious "spyware" to provide keystroke logs without timing information.

A tool similar to RUI was developed (Westerman et al., 1996) for the Windows 3.1 platform, but it appears no longer to be available. MICELAB (Baccino \& Kennedy, 1995) is another similar tool, but it does not run on modern computers, although Baccino and Kennedy's analysis approach is still helpful in analyzing mouse movements. Another tool, InputLogger, can be used to obtain user interactions across generic interfaces, but it works only on the Classic Macintosh (pre-Mac OS X) platform (Trewin, 1998). Several large commercial products are also available.

\section{Description of RUI}

RUI is a keystroke and mouse action logger for the Windows (2000 and XP) and Mac OS X (10.3 and later versions) platforms. RUI's user interface includes options for which types of actions to record, including keystrokes and mouse movements. The data collected is stored in a $\log$ file (see Figure 1) as a list of the timestamps, actions, and arguments (if any), such as key pressed or move location. To start and stop recording, the hot keys $C t r l+R$ and $C t r l+S$, respectively, are provided. The amount of data recorded with extensive mouse movements is about $22 \mathrm{~K} /$ min, but if the user moves the mouse continuously, the amount of data can be as high as $156 \mathrm{~K} / \mathrm{min}$. The timestamp for a single keystroke is approximately 19 characters, so less mouse-intensive activities will have correspondingly smaller log files.

The data collected can be replayed using the Replay option or played faster or slower than in real time. During 


\begin{tabular}{|c|c|c|c|}
\hline \multicolumn{4}{|c|}{ E er.txt - Notepad } \\
\hline File Edit Format & View Help & & \\
\hline $00: 00: 02: 874$ & Moved & 97 & 321 \\
\hline $00: 00: 02: 884$ & Moved & 97 & 319 \\
\hline $00: 00: 02: 894$ & Moved & 98 & 316 \\
\hline $00: 00: 02: 914$ & Moved & 98 & 315 \\
\hline $00: 00: 02: 954$ & Moved & 98 & 314 \\
\hline $00: 00: 02: 964$ & Moved & 98 & 313 \\
\hline $00: 00: 03: 575$ & Pressed & Left & \\
\hline $00: 00: 03: 835$ & Releasec & d Left & \\
\hline $00: 00: 04: 126$ & Moved & 98 & 311 \\
\hline $00: 00: 04: 136$ & Moved & 102 & 309 \\
\hline $00: 00: 04: 146$ & Moved & 112 & 303 \\
\hline $00: 00: 04: 166$ & Moved & 132 & 295 \\
\hline $00: 00: 04: 176$ & Moved & 168 & 279 \\
\hline $00: 00: 04: 196$ & Moved & 215 & 260 \\
\hline $00: 00: 04: 206$ & Moved & 265 & 242 \\
\hline $00: 00: 04: 216$ & Moved & 313 & 232 \\
\hline $00: 00: 04: 236$ & Moved & 349 & 230 \\
\hline $00: 00: 04: 246$ & Moved & 371 & 235 \\
\hline $00: 00: 04: 256$ & Moved & 389 & 245 \\
\hline $00: 00: 04: 276$ & Moved & 400 & 257 \\
\hline $00: 00: 04: 286$ & Moved & 406 & 269 \\
\hline $00: 00: 04: 296$ & Moved & 411 & 286 \\
\hline $00: 00: 04: 316$ & Moved & 411 & 299 \\
\hline $00: 00: 04: 326$ & Moved & 406 & 312 \\
\hline $00: 00: 04: 336$ & Moved & 400 & 324 \\
\hline $00: 00: 04: 356$ & Moved & 392 & 340 \\
\hline $00: 00: 06: 379$ & Key & $\mathrm{h}$ & \\
\hline $00: 00: 06: 509$ & Key & e & \\
\hline $00: 00: 06: 709$ & Key & 1 & \\
\hline $00: 00: 06: 860$ & Key & 1 & \\
\hline $00: 00: 07: 991$ & Key & o & \\
\hline
\end{tabular}

Figure 1. An example log of RUI showing the screen as the user moves the mouse, clicks, moves again, and then types "hello."

keystroke replay, if the observer wishes to see the same results occur in the interface, the system must be in the same state it was in before the recording started. Otherwise, the mouse moves and the keystrokes are created and performed by RUI, but the resulting effects are likely to be different. It is worth noting that very fast paced interfaces with variable system time lags may lead to difficulties with playback.

\section{Implementation of RUI for Windows}

RUI for Windows is developed in the .Net framework using C\#. One key feature of this application is that it uses events to capture keystrokes and mouse clicks. Thus, whenever there is a change in the state of the mouse buttons, in the position of the mouse, or in the state of any of the keys on the keyboard, an event is raised. To record mouse moves, a thread runs in the background, checking for any change in the position of the mouse. If there is such a change, a mouse move event is raised. The fastest rate at which RUI collects mouse movement data is every $10 \mathrm{msec}(100 \mathrm{~Hz})$. This rate can be lowered to create smaller log files.

Mouse activity is recorded with .Net using the MousePosition and MouseButton properties of Windows Forms. However, these forms will record activity only while the focus is on the application developed with
.Net. To record generic mouse activity during interaction with other applications, the Control.MouseButtons and Control.MousePosition classes are used. To capture global keystrokes, a Win32 API function, GetAsyncKeyState, is used. The System.DateTime function of the .Net framework is used to calculate the elapsed time between actions.

User actions are replayed using the log file, basing the mouse position on recorded values using the Cursor.Position function. To create mouse clicks and keystrokes on generic applications, mouse_event and keybd_event functions of the User32.dll are used.

\section{Implementation of RUI for the Macintosh}

RUI for Mac OS X is developed in C using the Carbon Event Manager APIs of the Carbon development framework. An event handler that listens for raw key down, mouse movement, and mouse click actions is installed and automatically called when such an event occurs. Inside the event handler routine, the event that occurred and its type are determined, and appropriate information similar to that used by RUI for Windows is logged into a text file. Playback of events is done using the CGPostKeyboardEvent and CGPostMouseEvent calls of the Quartz Services API, which provides access to low-level features of the Mac OS $\mathrm{X}$ window server.

\section{Use of RUI Data}

The data collected using RUI can thus be used to measure response times of users interacting with a wide range of interfaces and the time course of their behavior. In addition, information can be obtained about user interaction and the preferences for different input devices-for example, time to switch from mouse to keyboard - can also be determined. We describe an example application of RUI used with a complex interface.

Data collection in robot studies is a difficult task (Scholtz, Young, Drury, \& Yanco, 2004) and thus makes a good test environment. The Windows version of RUI was used to collect data from a human-robot interaction study.

RUI provides a rich set of data that has been successfully used to examine user interaction with the ER1 (www .evolution.com/er1/) robot's interface over sessions of up to $4 \mathrm{~h}$. (The robot interface, with its controls, is shown in Figure 2.) The ER1 is a simple, three-wheeled robot that carries a laptop as its controlling computer. The carried computer can drive the robot, or another computer can drive the robot through a remote wireless connection. Figure 2 illustrates a complex interface from which RUI can gather data. The data were studied using playback and other analysis tools and gave details about response times, numbers of errors, and even mouse-down durations across individuals performing a variety of tasks (Kukreja, 2004). The data collected by RUI helped to examine differences between novice and expert robot users and to understand several aspects of learning and visual memory. A cognitive model of user interaction was created in the ACT-R cognitive architecture (Anderson \& Lebiere, 1998) and vali- 


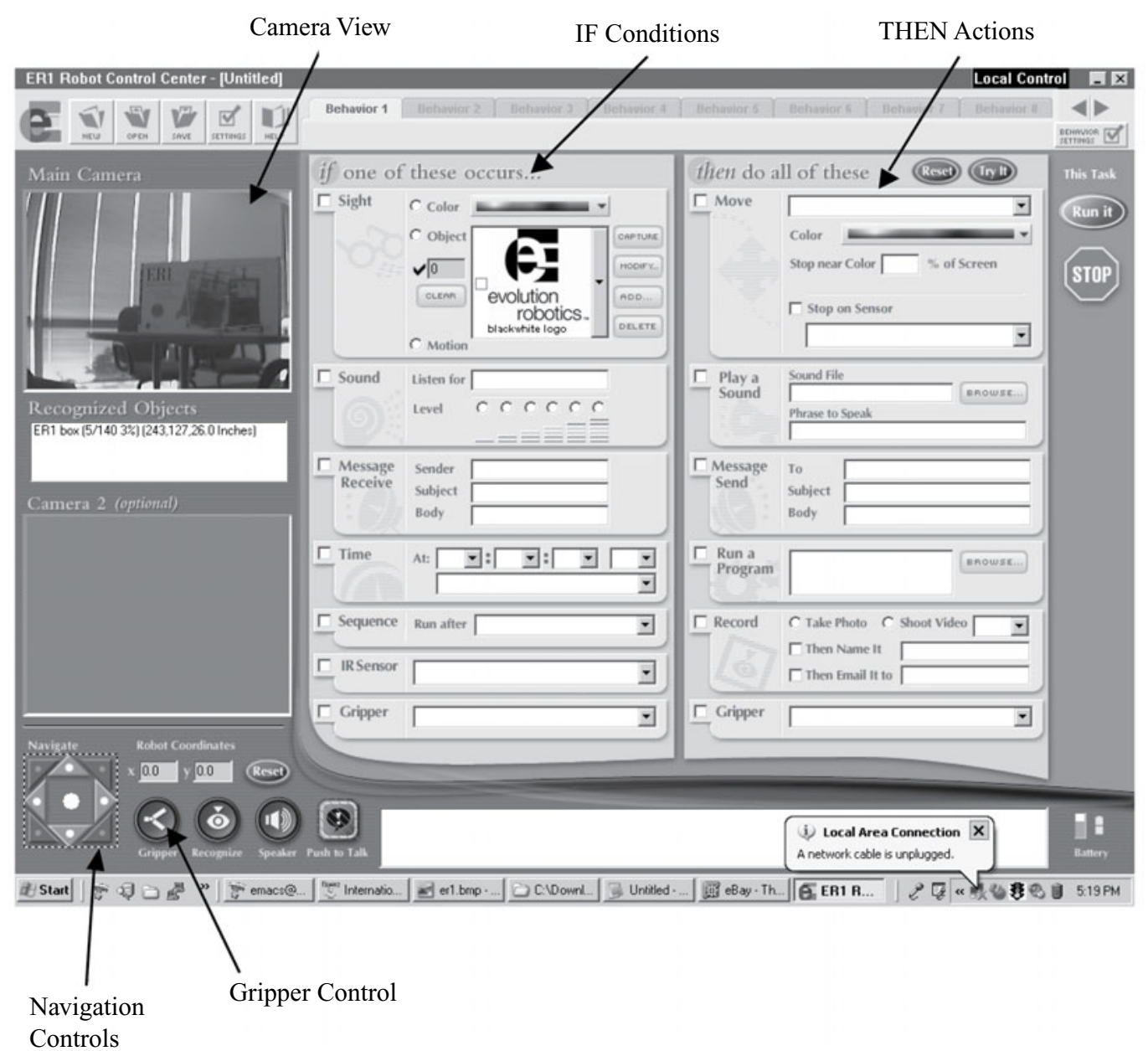

Figure 2. The ER1 robot interface.

dated against human data gathered using RUI (Kukreja, 2004; Ritter, Kukreja, \& St. Amant, in press).

A simple test of the quality of the timing data is shown in Figure 3. The histogram represents the times between actions (i.e., mouse movements, mouse clicks, and key- strokes), over a 15-min period, of a single user interacting with the ER1 human-robot interface.

The histogram shows that the distribution is right skewed, with the mean greater than the median and a long tail to the right, which is the usual case with response time

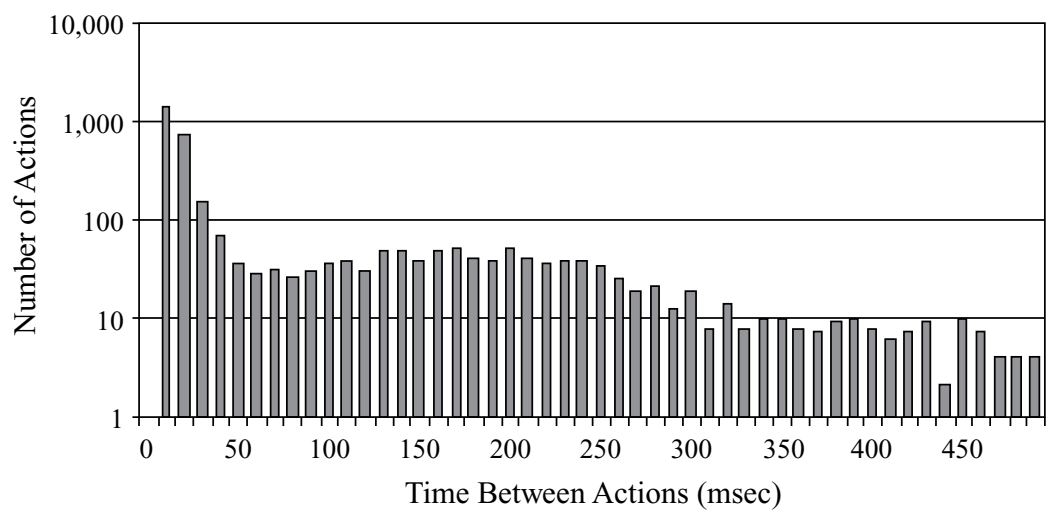

Figure 3. Histogram of the time between actions for $15 \mathrm{~min}$ of user behavior with the ER1 human-robot interface. 
data. Response time distributions should follow a positively skewed distribution, such as a gamma (or a Wald, Weibull, or ex-Gaussian distribution). Luce (1986), Van Zandt (1995), and Van Zandt and Ratcliff (1995), to note just a few reports, provide further discussions on this topic, some of them arguing for other, related distributions.

The distribution can be seen to approximate two gamma distributions. The first peak, at $10 \mathrm{msec}$, is for the mouse movement distribution, dominated by the 10-msec update during movements. The second, shallower peak, at around $175 \mathrm{msec}$, is for keystrokes.

Most importantly, a possible artifact that is missing is a spike representing 0 -msec response times. Such a spike would indicate that the timing program is not being called often enough and is batching the timestamping actions, although with rapid keystroke actions (which this data set does not include) simultaneous keypresses by both hands are possible but unlikely.

There could be other types of problems with RUI, but it passes these two tests related to the distribution of response times (gamma distribution and no spike at $0 \mathrm{msec}$ ). The use of the log scale helps show the keystroke and mouse click data without distortion by the frequent mouse movement data generated every $10 \mathrm{msec}$ when the mouse is moving. A similar curve is generated by the Macintosh version.

\section{Summary}

RUI provides a lightweight tool to record user behavior on Windows and Mac OS X machines. It records mouse movements, mouse buttonpresses, and keystrokes. The resulting logs are readable by both users and other software, and RUI can play the logs back for the analyst to see the details of the behavior as they emerge.

RUI has been used to study human-robot interaction, which was briefly described here as an example application. The resulting logs have been checked for correctness with two simple tests.

RUI provides a useful logging tool for researchers interested in recording behavior on most interfaces, because the interface does not have to be instrumented in order to study how users interact. Because it is available without cost and its source code is available on the Web, RUI might have a longer life than previous, comparable tools. Users are likely to find other uses for it. For example, the logs can be used for other analyses, such as plotting the path of a user's mouse and computing where the mouse is typically located.

\section{REFERENCES}

Anderson, J. R., \& LeBIERE, C. (1998). The atomic components of thought. Mahwah, NJ: Erlbaum.

BaCcino, T., \& KenNedy, A. (1995). MICELAB: Spatial processing of mouse movement in Turbo Pascal. Behavior Research Methods, Instruments, \& Computers, 27, 76-82.

Kukreja, U. (2004). Towards model-based evaluations of human-robot interfaces. Unpublished master's thesis, Pennsylvania State University, University Park, PA.

LuCE, R. D. (1986). Response times: Their role in inferring elementary mental organization. New York: Oxford University Press.

Ritter, F. E., Kukreja, U., \& St. Amant, R. (in press). Including a model of visual processing with a cognitive architecture to model a simple teleoperation task. Journal of Cognitive Engineering \& Decision Making.

Scholtz, J., Young, J., Drury, J. L., \& Yanco, H. A. (2004). Evaluation of human-robot interaction awareness in search and rescue. In Proceedings of the 2004 IEEE International Conference on Robotics and Animation (Vol. 3, pp. 2327-2332). Piscataway, NJ: IEEE Press.

TrewIN, S. (1998). InputLogger: General-purpose logging of keyboard and mouse events on an Apple Macintosh. Behavior Research Methods, Instruments, \& Computers, 30, 327-331.

VAN ZANDT, T. (1995). Analysis of response time distributions. In H. Pashler \& J. Wixted (Eds.), Stevens' Handbook of experimental psychology: Vol. 4. Methodology in experimental psychology (3rd ed., pp. 461-516). New York: Wiley.

VAN ZANDT, T., \& RATCLIFF, R. (1995). Statistical mimicking of reaction time data: Single-process models, parameter variability, and mixtures. Psychonomic Bulletin \& Review, 2, 20-54.

Westerman, S. J., Hambly, S., Alder, C., Wyatt-Millington, C. W., Shryane, N. M., Crawshaw, C. M., \& Hockey, G. R. J. (1996). Investigating the human-computer interface using the Datalogger. $\mathrm{Be}$ havior Research Methods, Instruments, \& Computers, 28, 603-606.

(Manuscript received September 24, 2004; revision accepted for publication August 31, 2005. ) 